\title{
VALORES HEMATOLOGICOS Y PARASITOLOGICOS DE BANDA NEGRA Myleus schomburgkii (PISCES, SERRASALMIDAE) CULTIVADOS EN ESTANQUES DE TIERRA
}

\author{
Christian FERNÁNDEZ-MÉNDEZ ${ }^{1}$,Anai GONZALES ${ }^{1,2}$, Gloria PIZANGO²
}

1 Instituto de Investigaciones de la Amazonía Peruana - IIAP, Programa para el Uso y Conservación del Agua y sus Recursos - AOUAREC, Carretera Iquitos Nauta km 4.5, Apartado 784, Iquitos, Perú. E-mail: cfernandez@iiap.org.pe

2 Universidad Nacional de la Amazonia Peruana (UNAP), Facultad de Ciencias Biológicas, Escuela de Acuicultura. Laboratorio de Hidrobiología. Iquitos, Perú.

\section{RESUMEN}

El objetivo de este estudio fue determinar los valores hematológicos e índices parasitarios de 25 juveniles de banda negra Myleus schomburgkii criados en condiciones de cautiverio en estanques de tierra bajo las siguientes condiciones: $29.3 \pm 0.9 \mathrm{C}^{\circ}, \mathrm{OD}: 3.5 \pm 1.2 \mathrm{mg} \cdot \mathrm{L}^{-1}$ y pH: $6.7 \pm 0.2$. Para la evaluación de cada ejemplar se tomó una muestra de sangre por punción de vena caudal e inmediatamente los animales fueron sacrificados para determinar el índice parasitario en base a la presencia de monogeneos en las branquias. Los parámetros sanguíneos se midieron empleando los métodos de microhematocrito y cianometahemoglobina. Los promedios de los parámetros hematológicos obtenidos muestran hematocrito (Hto): $37.17 \%$; hemoglobina (Hb): $10.34 \mathrm{~g} . \mathrm{dL}^{-1}$, eritrocitos $(\mathrm{Er}): 12.8 \times 10^{6} \cdot \mu \mathrm{L}^{-1}$ y leucocitos (Leu): $5.1 \times 10^{3} \mu . \mathrm{L}^{-1}$ de sangre. A nivel parasitológico, se identificaron dos géneros de la clase Monogenea (Notozothecium y Anacanthorus) infestando las branquias de los peces en estudio, con una prevalencia de $100 \%$ y abundancia media de 244 monogeneos.pez ${ }^{-1}$. Los resultados muestran el buen estado fisiológico de esta especie criada en condiciones normales de cautiverio.

PALABRAS CLAVE: Monogeneos, banda negra, Myleus schomburgkii, Amazonía, peces.

\section{HAEMATOLOGICAL AND PARASITOLOGICAL VALUES OF BLACK STRIPE Myleus schomburgkii (PISCES, SERRASALMIDAE) REARED IN PONDS}

\section{ABSTRACT}

The aim of this study was to determine the hematological and parasitological rates of 25 juvenile individuals of black stripe Myleus schomburgkii reared in earthen ponds under the following conditions: 29.3 $\pm 0.9^{\circ} \mathrm{C}$, DO: $3.5 \pm 1.2 \mathrm{mg} . \mathrm{L}^{-1} \mathrm{pH}: 6.7 \pm 0.2$. Blood collections were performed by tail vein puncture and the fish were sacrificed immediately to determine the presence of monogeneos in the gills. The blood parameters were measured using routine hematological techniques. The results show for Hematocrit (HCT): 37.17\%; Hemoglobin (Hb): 10.34 g.dL $\mathrm{L}^{-1}$, erythrocytes (Er): $12.8 \times 10^{6} . \mu \mathrm{L}^{-1}$ and leukocytes (Leu): $5.1 \times 10^{3} . \mu \mathrm{L}^{-1}$ blood. At the parasitological level, we identified two genera of the Monogenea class (Notozothecium and Anacanthorus) infesting the gills of the study fish, with 100\% prevalence, and mean abundance of 244 parasites.fish ${ }^{-1}$. Our results showed the good physiological status of this species reared in normal conditions of captivity.

KEYWORDS: Monogeneans, blach band myleus, Myleus schomburgkii Amazon, fish. 


\section{INTRODUCCIÓN}

Banda negra Myleus schomburgkii (Jardine \& Schomburgk, 1841) es un pez de agua dulce perteneciente a la familia Characidae. En el medio natural alcanza una talla de $35 \mathrm{~cm}$, diferenciándose de las demás especies de su género. Fue reportada para Perú en la cuenca del rio Nanay (Ortega et al., 2010). Esta especie tiene hábitos migratorios relacionados con la disponibilidad de alimento (macrófitas, zooplancton, semillas y frutos); se caracteriza por poseer una franja oscura transversal (Santos et al., 2006). Esta última característica la hace llamativa para la acuariofilia, cuya demanda actualmente es cubierta por la extracción en el medio natural, con el subsecuente deterioro de sus poblaciones naturales, debido a la fuerte presión de pesca sobre esta especie. Actualmente existe reducida información que sirva para el manejo sostenible de sus poblaciones naturales y del impacto causado por su sobreexplotación (CENDIPP, 2013). Una alternativa eficiente para disminuir la presión de extracción de los ambientes naturales sería desarrollar la piscicultura de esta especie. Para el desarrollo exitoso de la piscicultura se deben conocer los parámetros biológicos, fisiológicos, nutricionales, reproductivos, de sanidad, entre otros, para poder cultivar en cautiverio una determinada especie (Nuñez, 2009). De entre estos parámetros, la sanidad de la especie es un punto crucial para su mantenimiento en cautiverio, debido sobre todo a que los peces son fuertemente afectados por parásitos. Los monogeneos son los parásitos que se encuentran más comúnmente en los peces y son parásitos de gran especificidad de hospedero, es decir, cada especie de parasito está relacionado con una especie de pez (Centeno 2004; Aragort et al., 2002; Santos et al., 2013; Mathews et al., 2014). Los monogeneos presentan ciclo de vida directo, lo que favorece su transmisión entre los peces, llegando muchas veces a causar infestaciones elevadas (Shoolz \& Kuchta, 2005). Estos parásitos infestan los arcos branquiales del hospedero, con estructuras esclerotizadas presentes en su aparato de fijación (Thatcher, 2006), generando retardo en el crecimiento, disminución de peso y reducción de la tasa de fertilidad (Tavares et al., 2006). Cuando el equilibrio existente entre el ambiente y el patógeno se quiebra, surgen las epizootias que son de difícil control y normalmente llevan a los peces a la muerte (Malta et al., 2001), ocasionando grandes pérdidas económicas en los sistemas de producción piscícolas de todo el mundo (Flores \& Flores, 2003). Así mismo, estas enfermedades causadas por los parásitos pueden cambiar los valores hematológicos de los peces (Ranzani-Paiva et al., 2000), convirtiéndose el monitoreo de estos valores en una herramienta útil para medir el estado fisiológico de los peces en cultivo (Centeno et al., 2007), permitiendo realizar la identificación, diagnóstico y control de enfermedades como una herramienta de prevención (Mendoza et al., 2015).

Los pocos estudios sobre el cultivo en cautiverio de banda negra, están relacionados a la nutrición de esta especie (García-Ayala et al., 2012), no habiendo sido reportado en la literatura informaciones relacionadas a su reproducción y sanidad, aspectos que permitirían el desarrollo de su piscicultura. El presente estudio pretende contribuir al conocimiento de los parámetros de sanidad a través de la evaluación de los valores hematológicos e índices parasitarios de 25 juveniles de banda negra criados en estanques de tierra.

\section{MATERIALES Y MÉTODOS}

\section{Lugar de estudio, población y condiciones de cultivo}

El estudio se realizó en las instalaciones del Centro de Investigaciones Fernando Alcántara Bocanegra (CIFAB) del Instituto de investigaciones de la Amazonia Peruana (IIAP), localizado en la carretera Iquitos-Nauta, en la provincia de Maynas, Loreto, Perú.

Se utilizaron un total de 25 juveniles de banda negra con una longitud total promedio de $17.3 \pm 1.4$ $\mathrm{cm}$ y peso promedio de $115.9 \pm 20.8 \mathrm{~g}$, extraídos de un lote 100 especímenes cultivados en un estanque de tierra de $9 \mathrm{~m}^{2}$ de espejo de agua y de $60 \mathrm{~cm}$ de profundidad. Durante el tiempo que duro el estudio, los peces fueron alimentados dos veces al día $(8: 00$ y 16:00) con una dieta comercial extrusada con un tenor de $22 \%$ de proteína bruta. Los parámetros físico-químicos promedios del estanque fueron: temperatura $=29.3 \pm 0.9 \mathrm{C}$, oxígeno disuelto $=3.5 \pm$ $1.2 \mathrm{mg} . \mathrm{L}^{-1}, \mathrm{pH}=6.7 \pm 0.2$, estos parámetros fueron monitoreados tres veces por día con ayuda de un equipo multiparamétrico YSI y a través del kit limnológico de análisis de agua dulce (LaMotte AQ2).

\section{Toma de muestra sanguínea}

Las muestras de sangre se obtuvieron mediante punción de la arteria caudal a nivel del arco hemal utilizando jeringas desechables de $3 \mathrm{~mL}$, con anticoagulante EDTA al 10\%; inmediatamente la sangre extraída fue traspasada a tubos de $2 \mathrm{ml}$ y conservadas a $4^{\circ} \mathrm{C}$ para preservar la viabilidad de las células. 


\section{Análisis hematológico}

Se realizaron determinaciones de hematocrito por el método del microhematocrito, concentración de hemoglobina $(\mathrm{Hb})$ utilizando el método de la cianometahemoglobina y contaje de leucocitos y eritrocitos con una cámara de Neubauer (Blaxhall \& Daisley, 1973).

\section{Análisis parasitológico}

Los peces fueron sacrificados después de la extracción de sangre con un estilete a través de la punción en el cerebro. Se extrajo las branquias que fueron separadas y ubicadas en placas petri con agua destilada. Los monogeneos fueron extraídos y fijados de las branquias para el estudio de estructuras esclerotizadas en solución de amonio-picrato y glicerina (GAP) proporción 3:1 según Mamlberg (1957). La identificación de los parásitos fue en base a la metodología de Thatcher (2006) y Schoolz y Kuchta (2005). Los índices parasitarios calculados para evaluar el nivel de infestación de parásitos fueron: prevalencia (P) y abundancia media (AM) (Bush etal., 1997).

\section{Análisis de datos}

Los resultados de la evaluación parasitaria y hematológica se procesaron en hojas de Excel y analizaron usando ANOVA, para determinar la correlación se usó el coeficiente de Pearson con el software estadístico "Statgraphics Plus".

\section{RESULTADOS Y DISCUSIÓN}

La tabla 1 muestra los parámetros hematológicos de Myleus schomburgkii cultivados en estanques de tierra. Estos valores encontrados se encuentran cercanos a los obtenidos por otros estudios en carácidos amazónicos cultivados en estanques de tierra (Tavares-Dias et al., 1999; Soberon et al., 2007; Centeno et al., 2004). Los valores obtenidos en estos parámetros pueden ser considerados relativos, debido a que múltiples factores tanto ambientales (condiciones de cultivo), como propios del espécimen (nutrición, sexo, edad, entre otros) podrían causar variaciones en los parámetros hematológicos (Soberon et al., 2007). Así mismo, los valores de hematocrito pueden variar de acuerdo a la especie por familia, si es de cautiverio o del medio natural (Tavares-Dias \& Moraes, 2004). Tavares-Dias et al. (1998) reportaron para Colossoma macropomum, Piaractus mesopotamicus y Brycon cephalus valores de hematocrito entre 20.8 - $44 \%$; nosotros obtuvimos relativamente valores inferiores y superiores a los mencionados por estos autores, pero dentro de los estándares normales de referencia. Esto se debe probablemente a la competición intraespecífica por alimento a la que se someten los peces en situaciones de la alta densidad de crianza (Paredes et al., 2013), tal como ocurrió en el caso de los peces de nuestro estudio.

El factor de condición (k) y el hematocrito mostraron una relación lineal negativa $(\mathrm{P}<0.05)$ con $\mathrm{R}=0.556$ como se muestra en la Figura 1 . El hematocrito muestra el estado de condición fisiológica de los peces y es el determinante para el diagnóstico de anemia en peces (Tucker et al., 1989; Mendoza et al., 2015), mientras que el factor de condición $(\mathrm{K})$ muestra el bienestar en relación al peso/longitud (Froese, 2006). Estos resultados evidencian que cuando esta especie es cultivada en estanques de tierra, los individuos que tienen las mejores condiciones en peso respecto a la longitud poseen menor porcentaje de hematocrito.

Tabla 1. Valores hematológicos de juveniles de banda negra M. schomburgkii cultivados en estanques de tierra $(n=25)$.

\begin{tabular}{|c|c|c|c|}
\hline Parámetro & Promedio & $\begin{array}{c}\text { Desviación } \\
\text { estándar }\end{array}$ & Rango \\
\hline Hematocrito (\%) & 37.18 & 5.9 & $20.3-51.3$ \\
\hline Hemoglobina $\left(\mathrm{g} \cdot \mathrm{dL}^{-1}\right)$ & 10.34 & 5.9 & $1.2-31.7$ \\
\hline Eritrocitos $\left(10^{6} . \mu \mathrm{L}^{3}\right)$ & 12.8 & 12.4 & $4-63.3$ \\
\hline Leucocito $\left(10^{3} . \mu \mathrm{L}^{3}\right)$ & 5.1 & 2.8 & $0.8-14$ \\
\hline VCM (fl) & 37.5 & 19.5 & $6.7-86.7$ \\
\hline CM (pg.cel $\left.{ }^{-1}\right)$ & 8.9 & 6.3 & $1.3-31.8$ \\
\hline CHCM (\%) & 27.25 & 16.5 & $5.5-91.1$ \\
\hline
\end{tabular}


Uno de los factores de mayor influencia en los parámetros hematológicos es la parasitosis, por lo que un monitoreo constante de los peces en cultivo es necesario para mantener la sanidad del cultivo. Para determinar el grado de infección que estos parásitos causan, es necesario determinar los índices parasitarios, los cuales sirven para el análisis cuantitativo de los parásitos que son encontrados en un determinado pez (Bush et al., 1997). Según algunos autores, el alto índice parasitario dependerá de los factores ambientales del medio (Mancini et al., 2002, Modu et al., 2012 \& Martins et al., 2014).

Nuestros resultados muestran la presencia de dos géneros de monogeneos (Nothozothecium y Anacanthorus) en las branquias de los juveniles de Myleus schomburgkii. Estos parásitos fueron identificados en base a las estructuras esclerotizadas "opistohaptor" y el órgano copulador "cirrus". Los géneros Notozothecium y Anacanthorus muestran una variada habilidad para infestar especies hospederas íntimamente relacionadas (Fischer et al., 2003). Estos géneros fueron reportados parasitando a las especies Myleus schomburgkii, M. rubripinnus, M. pacu, M. rhomboidalis, Mylesinus paraschomburgkii, M. paucisquamatus, Piaractus brachypomum, P. mesopotamicus y Colossoma macropomum (Tavares-Dias et al., 2001; Aragort et al., 2002; Thatcher., 2006; Gonzales et al., 2015), especies hospederas muy próximas taxonómicamente, por lo que se podría afirmar que son parásitos que se especializan en parasitar a peces del orden Characiformes (Kritsky et al., 1992).

Notozothecium sp. y Anacanthorus sp. son parásitos que pertenecen a la familia Dactylogyridae, la cual según Thatcher (1991) presenta especies que prefieren parasitar las branquias de los peces, es decir, presentarían especificidad del lugar a parasitar en el hospedero.

Los valores de prevalencia (P) y abundancia media (AM) de los dos géneros de monogeneos identificados en las branquias se muestran en la Tabla 2; la prevalencia total y por cada especie de monogeneos fue del $100 \%$. La especie Notozothecium sp. fue la más abundante (226 parásitos.pez ${ }^{-1}$ ) lo que refleja la alta capacidad de infestación de este monogeneo en la población de banda negra. A pesar de la elevada prevalencia y abundancia de este género, no hubo mortalidad de los peces ni signos de enfermedad aparente, debido a que la salud de los peces posiblemente estuvo relacionada directamente con el equilibrio hospedero-parasito-ambiente. A menudo, estos tres factores interaccionan entre sí, para permanecer estables en el medio (Santos et al., 2013; Wilfrod, 1962 y Lambert, 1990). Sin embargo, el índice parasitario del género Anacanthorus mostró una elevada prevalencia (100\%), pero baja abundancia media (18 monogeneos.pez ${ }^{-1}$ ) en comparación con

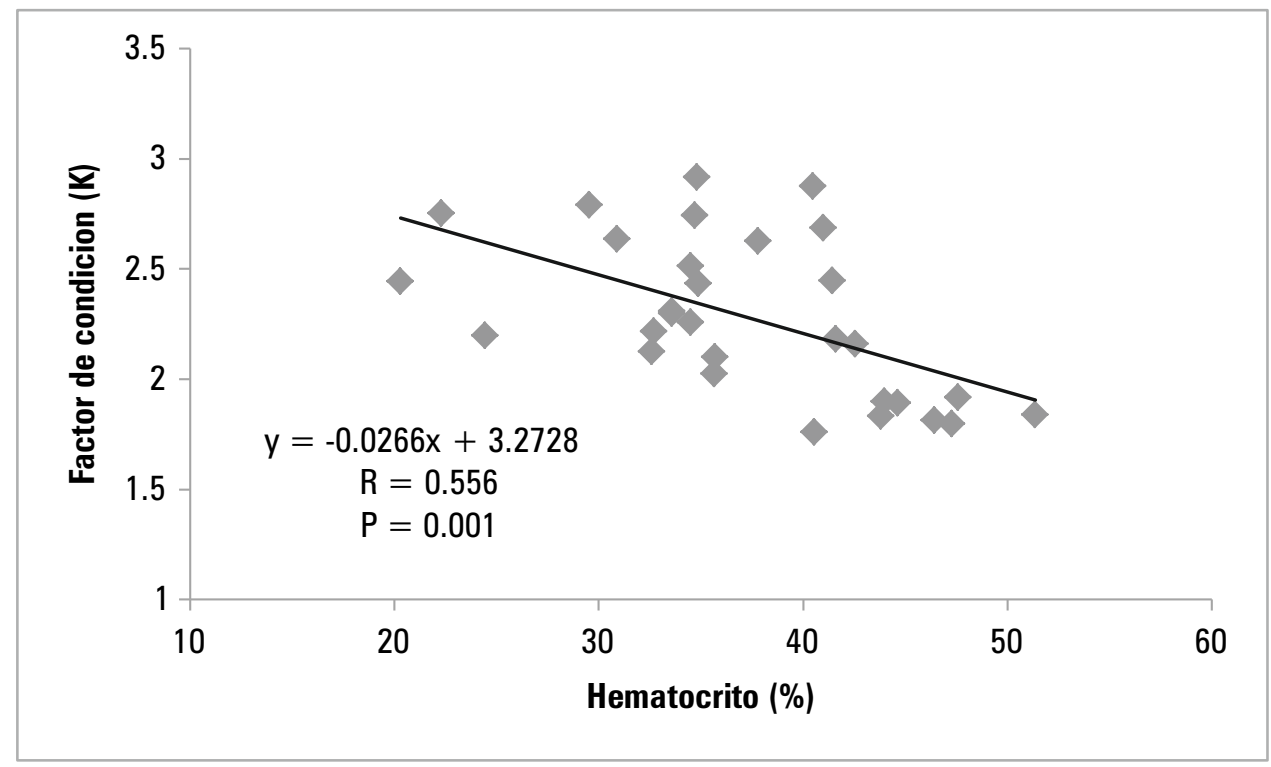

Figura 1. Correlación lineal $(P<0.05)$ entre el factor de condición y hematocrito de juveniles $(n=25)$ de banda negra M. schomburgkii cultivados en estanques de tierra. 
Tabla 2. Prevalencia (\%) y Abundancia media (AM) por género de monogeneos branquiales encontrados en los juveniles de banda negra $M$. schomburgkii cultivados en estanques de tierra $(\mathrm{n}=25)$.

PEZ/PARASITOS

\begin{tabular}{lcc}
\hline M. schomburgkii & $\mathrm{P}(\%)$ & AM (parasito.pez ${ }^{-1}$ ) \\
Clase & & \\
Monogenea & 100 & 244 \\
Género & & \\
Nothozhothecium & 100 & 226 \\
Anacanthorus & 100 & 18 \\
\hline
\end{tabular}

Notozothecium. Esto podría explicarse porque los parámetros hematológicos de nuestros peces no variaron, ya que Anacanthorus solo es considerado patógeno a elevados niveles de infestación, debido a que causa reducción en la capacidad respiratoria de los peces hospederos llevándolos a la mortalidad (Santos et al., 2013). Esto ya fue corroborado en Colossoma macropomum por Centeno et al. (2004), quien reportó este parasito en los peces examinados, constatando que los mismos no presentaron ningún signo o síntoma de enfermedades.

Los parámetros hematológicos de nuestros peces muestran que las condiciones de cultivo establecidas en este estudio, son las adecuadas para mantener un buen estado fisiológico de Myleus schomburgkii bajo condiciones de cautiverio.

\section{AGRADECIMIENTOS}

Los autores agradecen al Centro de Investigación y Promoción Popular (CENDIPP) por facilitar los peces para este estudio.

\section{BIBLIOGRAFÍA CITADA}

Aragort, W.; Morales, G.; León, E.; Pino, L. A.; Guillén, A.; Silva, M. 2002. Patologías asociadas a monogeneos branquiales en cachama bajo cultivo.Vet. Trop, 27(2), 75-85.

Blaxhall, P.C.; Daisley, K.W. 1973. Routine haematological methods for use with fish blood. Journal of Fish Biology, 5(6), 771-781.

Bush, A.O.; Lafferty, K.D.; Lotz, J. M.; Shostak, A. W. 1997. Parasitology meets ecology on its own terms: Margolis et al. revisited. The Journal of parasitology, 575-583.
Centeno, L.; Silva-Acuña, A.; Silva-Acuña, R.; Pérez, J.L. 2004. Fauna ectoparasitaria asociada a Colossoma macropomum y al híbrido de $C$. macropomum $x$ piaractus brachypomus, cultivados en el estado delta Amacuro, Venezuela. Bioagro, 16(2), 121-126.

Centeno, L.; Silva-Acuña, R.; Barrios, R.; Salazar Lugo, R.; Matute, C.; Pérez, J.L. 2007. Características hematológicas de la cachama (Colossoma macropomum) en tres etapas de crecimiento cultivadas en el estado Delta Amacuro, Venezuela. Zootecnia tropical, 25(4), 237-243.

Centro de investigación y promoción popular. 2013. Programa de manejo pesquero de Myleus schomburgkii "banda negra" y Myleus rubripinis "curuhuara" en el bajo rio Nanay (2014 - 2018). Iquitos. 93 pp.

Cohen, S.C.; Kohn, A. 2009. On Dactylogyridae (Monogenea) of four species of characid fishes from Brazil. Check List, 5(2), 351-356.

Fischer, C.; Malta, J.C.D.O.; Varella, A.M.B. 2003. The fauna of parasites of the tambaqui, Colossoma macropomum (Cuvier, 1818) (Characiformes: Characidae) from middle Solimões River and lower Amazonas River and their potential as biological indicators. Acta Amazonica, 33(4), 651-662

Froese, R. 2006. Cube law, condition factor and weight-length relationships: history, metaanalysis and recommendations. Journal of Applied Ichthyology, 22:241-253.

Gonzales, A.F.; Mathews, P.D.; Luna, L.E.; Mathews, J.D. 2015. Outbreak of Notozothecium bethae (Monogenea: Dactylogyridae) in Myleus schomburgkii (Actinopterygii: Characiformes) cultured in the Peruvian Amazon. Journal of Parasitic Diseases, 1-5. 
Kritsky, D.C.; Boeger, W.A.; Van Every, L.R. 1992. Neotropical Monogenoidea. 17. Anacanthorus Mizelle and Price, 1965 (Dactylogyridae, Anacanthorinae) from characoid fishes of the central Amazon. Journal of the Helminthological Society of Washington, 59(1), 25-51

Lambert, A.; El Gharbi, S. 1995. Monogenean host specificity as a biological and taxonomic indicator for fish. Biological Conservation, 72(2), 227-235.

Nuñez, J. 2009. Domesticación de nouvelles espèces d'intérêt piscicole en Amazonie. Cah Agric, $18(2-3)$

Mancini, M.; Rodríguez, C.; Finola, M.; Basualdo, C.; Prosperi, C. 2000. Mortandad de peces en un lago recreacional del Sur de Córdoba, Argentina. Revista Aquatic, 11, 1-7.

Martins, M.L.; de Sá, A.R.S.; Jerônimo, G.T.; Tancredo, K.R.; Gonçalves, E.L. T.; Bampi, D.; Sandin, A.M. 2014. Microhabitat preference and seasonality of Gill Monogeneans in Nile Tilapia reared in Southern Brazil. Neotropical Helminthology, 8(1), 47-58.

Mathews, P.D.; Malheiros, A.F.; Ismiño O.R.; Vasquez, N.D. 2014 Jainus amazonensis (Monogenea: Dactylogyridae) parasites of Brycon cephalus (Günther, 1869) cultured in the lowland of the Peruvian Amazon. Croat J Fish 72:83-86.

Malmberg, G. 1957. Om förekomsten av Gyrodactylus på svenska fiskar.Skrifter Utgivna av Södra Sveriges Fiskeriförening, Arsskrift, 1956, 19-76.

Mendoza, F.J.A.; Cantú, E.M.T.; Leal, J.M. 2015. Parámetros hemáticos en tres especies de peces (Ictalurus punctatus, Morone saxatilis y Micropterus salmoides) en condiciones de desnutrición. Revista Iberoamericana para la Investigación y el Desarrollo Docente, 3(6).

Modu, B.M.; Saiful, M.; Kartini, M.; Kasim, Z.; Hassan, M.; Shaharom-Harrison, F. M. 2012. Effects of Water Quality and Monogenean Parasite in the Gills of Freshwater Cat Fish, Hemibagrus nemurus Valenciennes 1840.

Paredes, D.; Alvarez, C.; Valencia, T. 2015. Caracterización hematológica y bioquímica de juveniles de Arapaima gigas "paiches" bajo condiciones de cultivo en selva alta. Revista Investigación y Amazonía, 3(2), 67-70.

Ranzani-Paiva, M.J.T.; Silva-Souza, A.T.; Pavanelli, G.C.; Takemoto, R.M. 2000. Hematological characteristics and relative condition factor $(\mathrm{Kn})$ associated with parasitism in Schizodon borellii (Osteichthyes, Anostomidae) and Prochilodus lineatus (Osteichthyes, Prochilodontidae) from Paraná river, Porto Rico region, Paraná, Brazil. Acta Scientiarum, 22(2), 515-521.

Santos, E.F.; Tavares-Dias, M.; Pinheiro, D.A.; Neves, L.R.; Marinho, R.D.G. B.; Dias, M.K.R. 2013. Fauna parasitária de tambaqui Colossoma macropomum (Characidae) cultivado em tanque-rede no estado do Amapá, Amazônia oriental. Acta Amazonica, 43, 107-114.

Soberon, L.; Mathews, P.; Malherios, A. 2014. Hematological parameters of Colossoma macropomum naturally parasitized by Anacanthorus spathulatus (Monogenea: Dactylogiridae) in fish farm in the Peruvian Amazon. International Aquatic Research, 6(4), 251-255.

Tavares-Dias, M.; Sandrim, E.F.S.; Sandrim, A. 1998. Características hematológicas do tambaqui (Colossoma macropomum) Cuvier, 1818 (Osteichthyes: Characidae) em sistema de monocultivo intensivo. I. Série Eritrocitária. Rev. Brasil. Biol, 58(2), 197-202.

Tavares-Dias, M.; Sandrim, E.F.S.; Campos-Filho, E.D. 1999. Características hematológicas do tambaqui Colossoma macropomum Cuvier (Osteichthyes: Characidae) em sistema de monocultivo intensivo. II. Revista Brasileira de Zoologia, 16, 175-184.

Tavares-Dias, M.; Moraes, F.R.; Martins, M.L.; Kronka, S.N. 2001. Parasitic fauna of cultivated fishes in feefishing farm of franca, state of São Paulo, Brazil. II. Metazoans. Revista Brasileira de Zoologia, 18, 81-95.

Tavares-Dias, M.; Moura, F.R.M. 2004. Hematologia de peixes teleósteos. 1.ed. Ribeirão Preto. 144pp.

Thatcher, V.E. 1991 Amazon Fish Parasites. Amazonia, 11(3-4), 263-571.

Tucker, C.S.; Francis-Floyd, R.; Beleau, M.H. 1989. Nitrite-induced anemia in channel catfish, Ictalurus punctatus Rafinesque. Bulletin of environmental contamination and toxicology, 43(2), 295-301.

Wilford, O.O. 1962. Animal parasites: Their biology and life cycles. University of Michigan, 346pp.

Recibido: 10 de setiembre del 2015

Aceptado para publicación: 28 de octubre del 2015 